\title{
The termination shock of a magnetar wind: a possible origin of gamma-ray burst X-ray afterglow emission
}

\author{
Z. $\mathrm{Mao}^{1,2}$, Y. W. Yu ${ }^{1,2,3}$, Z. G. Dai ${ }^{4}$, C. M. $\mathrm{Pi}^{5}$, and X. P. Zheng ${ }^{2}$ \\ ${ }^{1}$ Institute of Astrophysics, Huazhong Normal University, Wuhan 430079, PR China \\ e-mail: yuyw@phy.ccnu.edu.cn \\ 2 Key Laboratory of Quark and Lepton Physics (Huazhong Normal University), Ministry of Education, Wuhan 430079, PR China \\ Department of Physics, The University of Hong Kong, Hong Kong, PR China \\ 4 Department of Astronomy, Nanjing University, Nanjing 210093, PR China \\ 5 Department of Physics and Electronics, Hubei University of Education, Wuhan 430205, PR China
}

Received 7 September 2009 / Accepted 13 May 2010

\begin{abstract}
Context. Swift observations suggest that the X-ray afterglow emission of some gamma-ray bursts (GRB) may have internal origins, and the conventional external shock (ES) cannot be the exclusive source of the afterglow emission.

Aims. If the central compact objects of some GRBs are millisecond magentars, the magnetar winds could play an important role in the (internal) X-ray afterglow emission, which is our focus here.

Methods. The dynamics and the synchrotron radiation of the termination shock (TS) of the magmnetar winds, as well as the simultaneous GRB ES, are investigated by considering the magnetization of the winds.

Results. As a result of the competition between the emission of the wind TS and the GRB ES, two basic types of X-ray afterglows are predicted, i.e., the TS-dominated and the ES-dominated types. Moreover, our results also show that both of the two types of afterglows have a shallow-decay phase and a normal-decay one, as observed by the Swift satellite. This indicates that some observed X-ray afterglows could be (internally) produced by the magnetar winds, but not necessarily GRB ESs.
\end{abstract}

Key words. gamma rays: bursts - shock waves - stars: winds, outflows

\section{Introduction}

The conventional fireball model for gamma-ray bursts (GRBs; see reviews by Piran 2005; Mészáros 2006) suggests that the burst emission is produced by internal dissipations in a relativistic, expanding fireball, while the afterglow emission arises from the deceleration of the fireball in the circumburst medium by exciting an external shock (ES). However, Swift observations have showed that some X-ray afterglow emission components may sometimes also have internal origins. The most solid evidence of this is the rapid rise and decline of X-ray afterglow flares (e.g., Burrows et al. 2005; Yu \& Dai 2009). Meanwhile, the ES model may also be challenged by some plateau emission that is followed by an abrupt cutoff (Troja et al. 2007). These phenomena indicate that internal afterglow emission ${ }^{1}$ could be more usual than considered before, at least in the X-ray band, whereas the ES is only one choice among various afterglow origins. In this case, some observed chromatic breaks in the afterglow light curves may also become more understandable. The long-lasting internal emission strongly requires long-lasting activities (energy release) of GRB central compact objects after the bursts, and to a certain extent, spinning-down magnetars are very plausible candidates (e.g., Dai et al. 2006; Troja et al. 2007).

It has been proposed for nearly two decades that GRBs could be produced by spinning-down, millisecond magnetars

\footnotetext{
1 In this paper we use the word "internal afterglow emission" to generally refer to the emission from any sites inside the ES.
}

(Usov 1992; Paczynski 1992; Duncan \& Thompson 1992). In this case, the relativistic fireball responsible for a GRB could be associated with a neutrino-driven wind (Thompson 1994; Metzger et al. 2007; Bucciantini et al. 2007, 2009), with a magnetic reconnection-accelerated wind (Drenkhahn 2002; Drenkhahn \& Spruit 2002; Giannois 2008), or with hyperaccretion onto the magnetars (Zhang \& Dai 2008, 2009). Of more interest here, the postburst magnetars could still keep abundant rotational energy, although a considerable fraction of the energy has been expended on the bursts. With the spin-down of the magnetars, this remaining energy can be released and drive a continuous energy outflow, i.e., a postburst wind. In view of the huge energy carried by the wind, Yu et al. (2010) suggested that some observed X-ray afterglows could be directly contributed by such winds, according to a qualitative comparison between the model and the observations. However, in that work, no specific radiation mechanism was addressed for the wind emission besides introducing a free constant for the radiation efficiency of the winds.

In this paper we try to search for a possible specific mechanism for the wind emission. Following Dai (2004) and Yu \& Dai (2007), we suggest that the termination shock (TS) of a magnetar wind, which arises from the interaction of the wind with the GRB fireball and the surrounding medium, may play an important role in the wind emission. Based on a more careful consideration, we redescribe the emission from the wind TS and the GRB ES by considering (1) the existence of the TS after the spin-down timescale and (2) the magnetization of the wind. 


\section{The spin-down of a magnetar and the magnetar wind}

Some short-term and violent processes mean that a newly born magnetar could drive an energetic, relativistic, complicated outflow to produce a GRB. During the first tens of seconds after the birth of the magnetar, its spin evolution must therefore be very complicated. In contrast, on the relatively longer (afterglow) timescales concerned here, the spin-down of the magnetar would be mainly controlled by the electromagnetic torque (leading to an MHD outflow) and the torque connected with gravitational wave radiation (Zhang \& Mészáros 2001). For the latter, if the equatorial ellipticity of the magnetar is mainly determined by the stellar magnetic field, the energy losses through gravitational-quadrupole radiation would not be essential for the spin-down of the star unless the magnetic field is $>3 \times 10^{17} \mathrm{G}$ (Usov 1992).

Therefore, for a magnetar with a magnetic field of $\sim 10^{14-15} \mathrm{G}$, its postburst spin-down luminosity can be simply estimated by (Shapiro \& Teukolsky 1983)

$$
\begin{aligned}
L_{\mathrm{md}}(t) & =\frac{(2 \pi)^{4}}{6 c^{3}} \frac{B_{\mathrm{s}}^{2} R_{\mathrm{s}}^{6}}{P_{\mathrm{s}}^{4}} \\
& \simeq 10^{47} \operatorname{erg~s}^{-1} R_{\mathrm{s}, 6}^{6} B_{\mathrm{s}, 14}^{2} P_{\mathrm{s}, \mathrm{i},-3}^{-4}\left(1+\frac{t}{T_{\mathrm{m}}}\right)^{-2},
\end{aligned}
$$

where $R_{\mathrm{s}, 6}=R_{s} / 10^{6} \mathrm{~cm}, B_{\mathrm{s}, 14}=B_{\mathrm{s}} / 10^{14} \mathrm{G}$, and $P_{\mathrm{s}, \mathrm{i},-3}=$ $P_{\mathrm{s}, \mathrm{i}} / 1 \mathrm{~ms}$ are the radius, magnetic field strength, and initial spin period of the magnetar, respectively. The characteristic spindown timescale reads as

$T_{\mathrm{m}}=\frac{6 c^{3}}{(2 \pi)^{2}} \frac{I_{\mathrm{s}} P_{\mathrm{s}, \mathrm{i}}^{2}}{B_{\mathrm{s}}^{2} R_{\mathrm{s}}^{6}} \simeq 2 \times 10^{5} \mathrm{~s} I_{\mathrm{s}, 45} R_{\mathrm{s}, 6}^{-6} B_{\mathrm{s}, 14}^{-2} P_{\mathrm{s}, \mathrm{i},-3}^{2}$,

where $I_{\mathrm{s}, 45}=I_{\mathrm{s}} / 10^{45} \mathrm{~g} \mathrm{~cm}$ is the stellar moment of inertial. The time $t$ is defined in the observer's frame, but the time zero is actually not strictly set at the GRB trigger time since Eq. (1) is only valid about $10-100 \mathrm{~s}$ after the birth of the magnetar. In other words, $t=0$ in this paper approximately represents the transition time from the prompt phase to the afterglow phase. Such a shift in the time zero would not influence the calculation of the afterglow emission on much longer timescales $\left(t>10^{2-3} \mathrm{~s}\right)$.

With the release of the stellar rotational energy after the burst, an MHD outflow is produced whose luminosity is determined by the spin-down luminosity as shown in Eq. (1). Unlike the GRB fireball that could be a neutrino-driven wind, this postburst outflow is likely to be initially Poynting flux-dominated, mixed by an electron-positron plasma. Since the fluctuating component of the magnetic fields in the outflow can in principle be dissipated by magnetic reconnection, the associated leptons are accelerated and the outflow gradually becomes an ultrarelativistic kinetic-energy flow consisting mainly of electronpositron plasma (lepton-dominated wind; Coroniti 1990). For the bulk Lorentz factor of the accelerated wind, we here arbitrarily take $\Gamma_{\mathrm{w}} \sim 10^{4}$ as a fiducial value following Atoyan (1999), who argues that the Crab pulsar wind had Lorentz factor $\Gamma_{\mathrm{w}} \sim 10^{4-7}$ to interpret the measured radio spectrum of the Crab Nebula.

\section{The dynamics}

Owing to its ultrarelativistic velocity, the magnetar wind catches up to and collide with the decelerating GRB ejecta. The interaction of the wind with the ejecta and the surrounding medium gives rise to a relativistic wind bubble, which includes four different regions separated by a contact discontinuity surface and two shocks (i.e., the TS of the wind that propagates into the cold wind and the GRB ES that propagates into the surrounding medium ${ }^{2}$ ). To be specific, the four regions are (1) unshocked medium; (2) shocked medium including the GRB ejecta; (3) shocked wind; and (4) unshocked cold wind close to the front of the TS. The shocked regions are emission regions.

We denote some quantities of region $i$ as follows: $n_{i}^{\prime}$ is the particle (proton or electron) number density, $B_{i}^{\prime}$ the magnetic field strength, $e_{i}^{\prime}$ the internal energy density, $P_{i}^{\prime}$ the pressure, where the primes refer to comoving frame, and the subscript $i$ represents regions $1-4 . \Gamma_{i}, \beta_{i}=\left(1-\Gamma_{i}^{-2}\right)^{1 / 2}$, and $u_{i}=\beta_{i} \Gamma_{i}$ are the bulk Lorentz factor, velocity, and four-velocity, respectively, measured in the local medium's rest frame. It is convenient to define a parameter

$\sigma \equiv \frac{B_{4}^{\prime 2}}{4 \pi n^{\prime}{ }_{4} m_{\mathrm{e}} c^{2}}$

to denote the degree of magnetization of the unshocked wind near the front of the TS, where $m_{\mathrm{e}}$ is the electron rest mass and $c$ the speed of light. By assuming the wind is isotropic, the comoving electron density of the unshocked wind can be expressed as $n_{4}^{\prime}=L_{\mathrm{md}} /\left[4 \pi R^{2} \Gamma_{4}^{2} m_{\mathrm{e}} c^{3}(1+\sigma)\right]$ with $\Gamma_{4}=\Gamma_{\mathrm{w}}$, and $R$ is the radius of the bubble in the thin shell approximation ${ }^{3}$. As considered in Zhang \& Kobayashi (2004) and Fan et al. (2004), a possible slow decrease in $B_{4}^{\prime 2}$ at large radii due to magnetic reconnection can be ignored. Thus same as $n_{4}^{\prime}, B_{4}^{\prime 2}$ would approximatively decrease as $R^{-2}$ with the expansion of the bubble, and thus the parameter $\sigma$ can be regarded as a constant.

To connect the properties of the two sides of the shocks, some shock jump conditions need to be listed as (Kennel \& Coroniti 1984; Zhang \& Kobayashi 2004)

$$
\begin{aligned}
\frac{e_{2}^{\prime}}{n_{2}^{\prime} m_{\mathrm{p}} c^{2}} & =\Gamma_{2}-1, \\
\frac{n_{2}^{\prime}}{n_{1}^{\prime}} & =4 \Gamma_{2}+3, \\
\frac{e_{3}^{\prime}}{n_{3}^{\prime} m_{\mathrm{e}} c^{2}} & =\left(\Gamma_{34}-1\right) f_{\mathrm{a}}, \\
\frac{n_{3}^{\prime}}{n_{4}^{\prime}} & =\left(4 \Gamma_{34}+3\right) f_{\mathrm{b}}, \\
\frac{B_{3}^{\prime}}{B_{4}^{\prime}} & =\left(4 \Gamma_{34}+3\right) f_{\mathrm{b}}, \text { for } \sigma>0
\end{aligned}
$$

where an adiabatic index of $4 / 3$ is adopted, $m_{\mathrm{p}}$ is the proton rest mass, $\Gamma_{34}=\left(\Gamma_{3} / \Gamma_{4}+\Gamma_{4} / \Gamma_{3}\right) / 2 \approx \Gamma_{4} /\left(2 \Gamma_{3}\right)$ is the bulk Lorentz factor of region 3 measured in the rest frame of region 4 ,

$$
\begin{aligned}
& f_{\mathrm{a}}=1-\frac{\left(\Gamma_{34}+1\right) \sigma}{2\left[\Gamma_{34} u_{3 t}^{2}+\left(\Gamma_{34}^{2}-1\right)^{1 / 2} u_{3 t}\left(u_{3 t}^{2}+1\right)^{1 / 2}\right]} \\
& f_{\mathrm{b}}=\frac{\Gamma_{34} u_{3 t}+\left(\Gamma_{34}^{2}-1\right)^{1 / 2}\left(u_{3 t}^{2}+1\right)^{1 / 2}}{\left(4 \Gamma_{34}+3\right) u_{3 t}}
\end{aligned}
$$

2 The initially-formed reverse and forward shocks in the GRB ejecta would cross the ejecta very quickly ( $\sim 100$ s), so we do not consider the effect of these two shocks.

${ }^{3}$ Following Eq. (7) in Dai (2004), the thickness of the relativistic wind bubble, $\Delta=R\left[\left(1+\frac{1}{8 \Gamma_{2}^{2}}\right)\left(1-\frac{\chi}{1+8 \Gamma_{2}^{2}}\right)-1\right] \ll R$, could be negligible with respect to the bubble radius, where the similarity variable $\chi$ is defined in Eq. (15). 
and $u_{3 t}$ is the four-velocity of region 3 measured in the rest frame of the TS. When $\Gamma_{34} \gg 1$, which is easily satisfied in this model (Yu \& Dai 2007), Eqs. (9) and (10) can be simplified as

$$
\begin{aligned}
& f_{\mathrm{a}} \approx 1-\frac{\sigma}{2\left[u_{3 t}^{2}+u_{3 t}\left(u_{3 t}^{2}+1\right)^{1 / 2}\right]}, \\
& f_{\mathrm{b}} \approx \frac{1}{4}\left[1+\left(1+\frac{1}{u_{3 t}^{2}}\right)^{1 / 2}\right],
\end{aligned}
$$

and $u_{3 t}$ can be solved analytically from $8(\sigma+1) u_{3 t}^{4}-\left(8 \sigma^{2}+10 \sigma+\right.$ 1) $u_{3 t}^{2}+\sigma^{2}=0$ (Zhang \& Kobayashi 2004).

Following Eq. (4), the total kinetic energy of region 2 can be calculated as $E_{\mathrm{k}, 2}=\left(\Gamma_{2}-1\right)\left(m_{\mathrm{ej}}+m_{\mathrm{sw}}\right) c^{2}+\Gamma_{2}\left(\Gamma_{2}-1\right) m_{\mathrm{sw}} c^{2}$, where $m_{\mathrm{ej}}$ and $m_{\mathrm{sw}}$ are the rest masses of the GRB ejecta and swept-up medium, respectively. Energy conservation requires that any increase in $E_{\mathrm{k}, 2}$ should be equal to the work done by region 3, i.e.,

$\mathrm{d} E_{\mathrm{k}, 2}=\delta W=4 \pi R^{2}\left(P_{\mathrm{th}, 3}^{\prime}+P_{B, 3}^{\prime}\right) \mathrm{d} R$,

where the thermal and magnetic components of the pressure of region 3 can be respectively calculated by $P_{\mathrm{th}, 3}^{\prime}=\frac{1}{3} e_{3}^{\prime}=$ $\frac{4}{3} \Gamma_{34}^{2} n_{4}^{\prime} m_{\mathrm{e}} c^{2} f_{\mathrm{a}} f_{\mathrm{b}}$ and $P_{B, 3}^{\prime}=B_{3}^{\prime 2} /(8 \pi)=8 \Gamma_{34}^{2} n_{4}^{\prime} m_{\mathrm{e}} c^{2} \sigma f_{\mathrm{b}}^{2}$ according to the shock jump conditions. Substituting the expression of $E_{\mathrm{k}, 2}$ into Eq. (13), we can obtain

$\frac{\mathrm{d} \Gamma_{2}}{\mathrm{~d} R}=\frac{4 \pi R^{2}\left[\left(P_{\mathrm{th}, 3}^{\prime}+P_{B, 3}^{\prime}\right) / c^{2}-\left(\Gamma_{2}^{2}-1\right) n_{1} m_{\mathrm{p}}\right]}{m_{\mathrm{ej}}+2 \Gamma_{2} m_{\mathrm{sw}}}$.

Meanwhile, the relationships between the properties of the two sides of the contact discontinuity surface show (Blandford \& McKee 1976)

$\Gamma_{3}=\Gamma_{2} \chi^{-1 / 2}$,

$P_{3}^{\prime}=P_{\mathrm{th}, 3}^{\prime}+P_{B, 3}^{\prime}=P_{2}^{\prime} \chi^{-17 / 12}$,

where the similarity variable can be solved to be

$\chi=\left[\frac{L_{\mathrm{md}}\left(f_{\mathrm{a}} f_{\mathrm{b}}+6 \sigma f_{\mathrm{b}}^{2}\right)}{16 \pi R^{2} \Gamma_{2}^{4} n_{1} m_{\mathrm{p}} c^{3}(1+\sigma)}\right]^{-12 / 29}$.

For a closing dynamic equation set, we also introduce another two equations in order to calculate the increasing masses of regions 2 and 3 as

$\frac{\mathrm{d} m_{\mathrm{sw}}}{\mathrm{d} R}=4 \pi R^{2} n_{1} m_{\mathrm{p}}$

and (Dai \& Lu 2002)

$\frac{\mathrm{d} m_{3}}{\mathrm{~d} R}=4 \pi R^{2}\left(\frac{\Gamma_{34} \beta_{34}}{\Gamma_{3} \beta_{3}}\right) n_{4}^{\prime} m_{\mathrm{e}}$.

Finally, we would like to pointed out that, in Dai (2004) and Yu \& Dai (2007), the authors only took the existence of the TS before $T_{\mathrm{m}}$ into account since the shallow decay afterglow phase during $\sim 10^{3-5} \mathrm{~s}$ was mainly concerned there. In contrast, here such an artificial cutoff of the TS has been abandoned for a more general investigation.

Combining the above dynamic equations with the relationships of $\mathrm{d} t=\left(1-\beta_{3}\right) \mathrm{d} R /\left(\beta_{3} c\right)$ for region 3 and $\mathrm{d} t=(1-$ $\left.\beta_{2}\right) \mathrm{d} R /\left(\beta_{2} c\right)$ for region 2 , we can numerically calculate the temporal evolution of the shock dynamics. As mentioned in Sect. 2, since $t=0$ is set at the transition time from the prompt phase to the afterglow phase, the initial conditions for our calculation is taken as $R_{\mathrm{i}}=10^{16} \mathrm{~cm}, \Gamma_{2, \mathrm{i}}=150$, and $E_{\mathrm{k}, 2, \mathrm{i}}=10^{51} \mathrm{erg}$, which

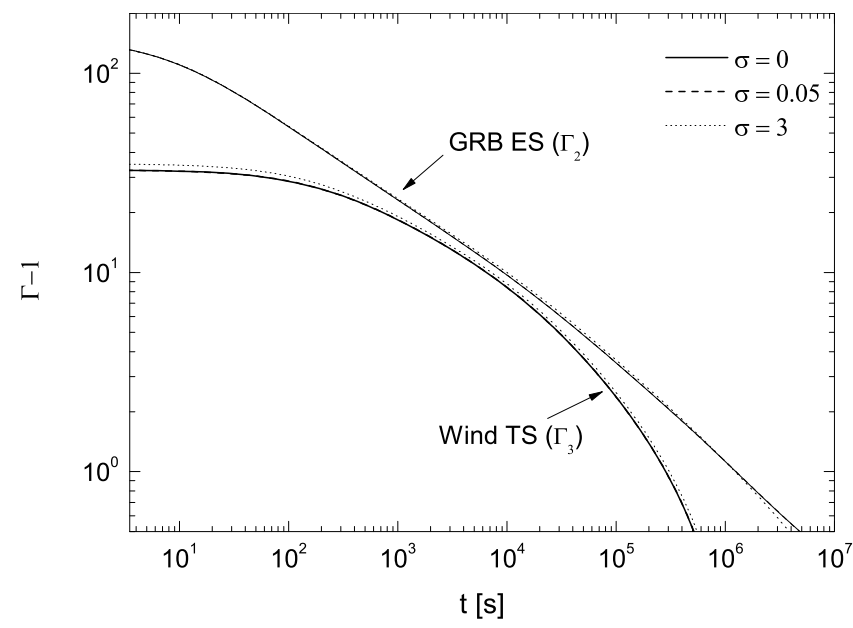

Fig. 1. The evolution of the bulk Lorentz factors of the GRB ES $\left(\Gamma_{2}\right)$ and the wind TS $\left(\Gamma_{3}\right)$ with different values of $\sigma$ as labeled. The parameters for the magnetar are as follows: $R_{\mathrm{s}, 6}=1, P_{\mathrm{s}, \mathrm{i},-3}=3, I_{\mathrm{s}, 45}=1, B_{\mathrm{s}, 14}=10$.

correspond to the deceleration timescale of the GRB ejecta. Figure 1 shows the evolution of the bulk Lorentz factors of the wind TS and the GRB ES with different values of $\sigma$. The nearly overlapping of the curves with $\sigma$ varying from 0 to 3 indicates that the dynamic evolutions of both the shocks are insensitive to the degree of magnetization of the wind. It is not surprising to obtain such a result, which actually has been know for a long time for ordinary pulsar wind nebulae (e.g., Emmering \& Chevalier 1987; Bucciantini et al. 2003; Del Zanna et al. 2004).

\section{The radiation from the shocks}

With the propagation of the shocks, the bulk kinetic energy of the GRB ejecta and the magnetar wind would be gradually converted into internal energy of the shocked materials. As usual, we assume that the internal energy of the shocked medium is shared by magnetic fields, electrons, and protons with fractions $\epsilon_{B}, \epsilon_{\mathrm{e}} \sim \sqrt{\epsilon_{B}}$ (Medvedev 2006) and $1-\epsilon_{\mathrm{e}}-\epsilon_{B}$, respectively. For the shocked wind, the energy fractions of the leptons and magnetic fields can be determined by the shock jump condition with a certain $\sigma$. Considering the shock acceleration of charge particles, the electrons in shocked regions are assumed to distribute as $n_{\gamma^{\prime}, i}^{\prime} \propto \gamma_{i}^{\prime-p}$ with a minimum electron Lorentz factor: $\gamma_{\mathrm{m}, 2}^{\prime}=\epsilon_{\mathrm{e}} g_{\mathrm{p}}\left(m_{\mathrm{p}} / m_{\mathrm{e}}\right)\left(\Gamma_{2}-1\right)$ for region 2 and $\gamma_{\mathrm{m}, 3}^{\prime}=$ $g_{\mathrm{p}} e_{3}^{\prime} /\left(n_{3}^{\prime} m_{\mathrm{e}} c^{2}\right)$ for region 3 with $g_{\mathrm{p}} \equiv(p-2) /(p-1)$. Owing to the synchrotron cooling of the electrons, a cooling Lorentz factor $\gamma_{c, i}^{\prime}=6 \pi m_{\mathrm{e}} c /\left(\sigma_{T} B_{i}^{\prime 2} \Gamma_{i} t\right)$ should also be defined by equaling the cooling time to the dynamic time, where $\sigma_{T}$ is Thomson cross section. Characterized by $\gamma_{\mathrm{m}}^{\prime}, \gamma_{\mathrm{c}}^{\prime}$ and $p$ (the subscript $i$ is omitted hereafter), a quasi-static distribution of the electrons can be written as (Huang et al. 2000)

$n_{\gamma^{\prime}}^{\prime} \propto \begin{cases}\left(\frac{\gamma^{\prime}}{\gamma_{\mathrm{L}}^{\prime}}\right)^{-x}, & \gamma_{\mathrm{L}}^{\prime} \leq \gamma^{\prime} \leq \gamma_{\mathrm{H}}^{\prime}, \\ \left(\frac{\gamma_{\mathrm{H}}^{\prime}}{\gamma_{\mathrm{L}}^{\prime}}\right)^{-x}\left(\frac{\gamma^{\prime}}{\gamma_{\mathrm{H}}^{\prime}}\right)^{-p-1}, & \gamma_{\mathrm{H}}^{\prime}<\gamma^{\prime} \leq \gamma_{M}^{\prime},\end{cases}$

where $\gamma_{\mathrm{H}}^{\prime}=\max \left(\gamma_{\mathrm{m}}^{\prime}, \gamma_{\mathrm{c}}^{\prime}\right), \gamma_{\mathrm{L}}^{\prime}=\min \left(\gamma_{\mathrm{m}}^{\prime}, \gamma_{\mathrm{c}}^{\prime}\right)$, and $x=2$ for $\gamma_{\mathrm{c}}^{\prime} \leq \gamma_{\mathrm{m}}^{\prime}$ and $x=p$ for $\gamma_{\mathrm{c}}^{\prime}>\gamma_{\mathrm{m}}^{\prime} \cdot \gamma_{M}^{\prime} \sim q_{\mathrm{e}} B^{\prime} R /\left(m_{\mathrm{e}} c^{2}\right)$ is the maximum electron Lorentz factor with $q_{\mathrm{e}}$ the electron charge.

For the X-ray band of interest in this paper, we only consider the synchrotron radiation of the electrons in both regions 2 


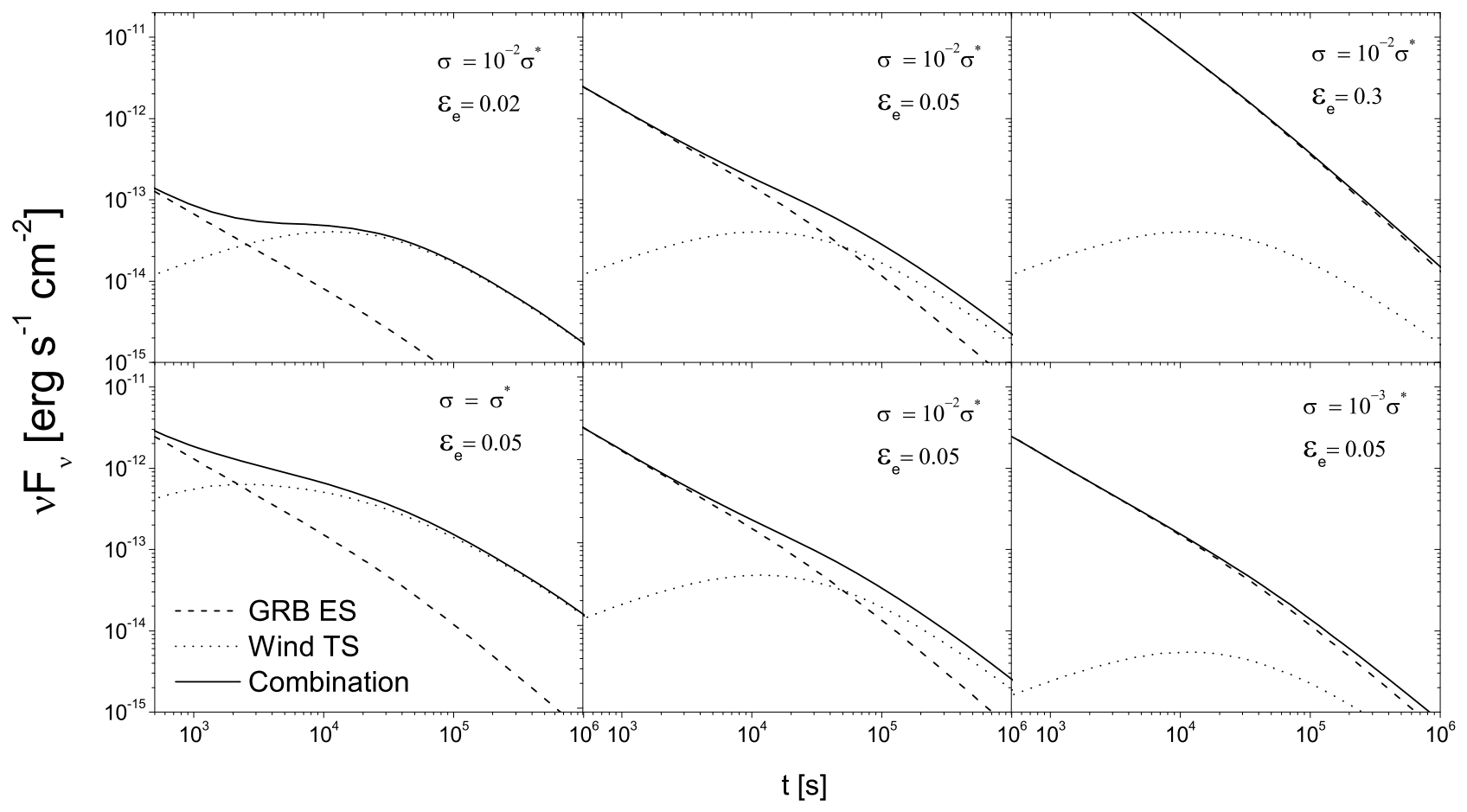

Fig. 2. X-ray (1 keV) light curves of the GRB ES (dashed lines) and the wind TS (dotted lines) as well as their combination (solid lines). The values of $\epsilon_{\mathrm{e}}$ and $\sigma$ are as labeled and $p=2.5$. The other model parameters are the same as those in Fig. 1.

and 3. The synchrotron emission coefficient at frequency $v^{\prime}$ can be given by (Rybicki \& Lightman 1979)

$j_{\nu^{\prime}}^{\prime}=\frac{1}{4 \pi} \int_{\gamma_{\mathrm{L}}^{\prime}}^{\gamma_{M}^{\prime}} n^{\prime}{ }_{\gamma^{\prime}}\left[\frac{\sqrt{3} q_{\mathrm{e}}^{3} B^{\prime}}{m_{\mathrm{e}} c^{2}} \frac{v^{\prime}}{v^{\prime}} \int_{\frac{v^{\prime}}{\nu_{0}}}^{\infty} K_{\frac{5}{3}}(y) \mathrm{d} y\right] \mathrm{d} \gamma^{\prime}$,

where $v_{0}^{\prime}=3 q_{\mathrm{e}} B^{\prime} \gamma^{\prime 2} /\left(4 \pi m_{\mathrm{e}} c\right), K_{\frac{5}{3}}(y)$ is the Bessel function. Then by assuming the viewing angle is zero, we can calculate the synchrotron flux density received by the observers as ( $\mathrm{Yu}$ et al. 2007)

$F_{\nu}(t)=\frac{1}{d_{\mathrm{L}}^{2}} \int_{0}^{\pi / 2} \frac{j^{\prime}{ }_{{ }^{\prime}} V^{\prime}}{\Gamma^{3}(1-\beta \cos \theta)^{3}} \frac{\sin \theta}{2} \cos \theta \mathrm{d} \theta$,

where $d_{\mathrm{L}} \sim 10^{28} \mathrm{~cm}$ is the luminosity distance of the GRB and $V^{\prime}$ is the comoving volume of the emission region. Considering the equal-arrival-time effect for a fixed observer's time $t$, different angles $\theta$ should correspond to different radii $R$, satisfying (Huang et al. 2000)

$t=\int_{0}^{R} \frac{1-\beta(r) \cos \theta}{\beta(r) c} \mathrm{~d} r$.

Therefore, as functions of radius, $j_{v^{\prime}}^{\prime}$ and $V^{\prime}$ should also be functions of $\theta$ in the integral in Eq. (22).

Some X-ray $(\sim 1 \mathrm{keV})$ light curves contributed by the wind TS and the GRB ES as well as the combination of them are shown in Fig. 2 with different values of $\sigma$ and $\epsilon_{\mathrm{e}}$. These two parameters control the energy densities of the electrons and magnetic fields, and thus determine the radiation efficiency of the two emission regions. Figure 2 shows that the emission from the ES is mainly dependent on the value of $\epsilon_{\mathrm{e}}$ (upper panel) but insensitive to $\sigma$ (lower panel). This is because the variation in $\sigma$ has little influence on the shock dynamics. In contrast, the lower panel of Fig. 2 shows that the emission from the TS is strongly dependent on the value of $\sigma$. More specifically, because a weak magnetic field can suppress the synchrotron radiation and a strong

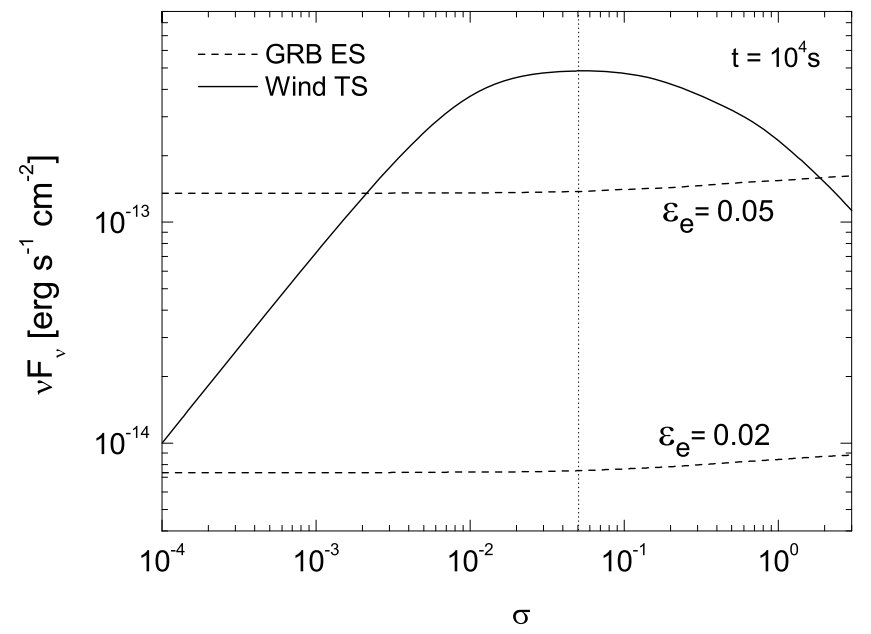

Fig. 3. The $\sigma$-dependence of the synchrotron flux of the GRB ES (dashed line) and the wind TS (solid line) at the time of $10^{4} \mathrm{~s}$. The vertical dotted line represents $\sigma^{*}=0.05$. The model parameters are the same as those in Fig. 2.

magnetic field reduces the energy fraction of the electrons, neither too low nor too high $\sigma$ leads to strong TS emission, which can be seen clearly in Fig. 3. For the model parameters adopted in Fig. 3, $\sigma=\sigma^{*} \approx 0.05$ results in the strongest TS emission. When the value of $\sigma$ is much closer to $\sigma^{*}$ or $\epsilon_{\mathrm{e}}$ is sufficiently small (e.g., $\epsilon_{\mathrm{e}} \sim 0.02$ ), the TS emission is very easy to exceed the ES emission. As a result, three types of X-ray afterglows can be predicted, i.e., the TS-dominated type, the ES-dominated type and the intermediate type, as shown by the left, right, and middle panels of Fig. 2, respectively. Qualitatively, such a result is basically consistent with the conjecture proposed by Yu et al. (2010).

As found previously (e.g., Dai 1998a,b; Zhang \& Mészáros 2001), the profile of the ES-dominated afterglow light curves 
(e.g., the cases in the right panel of Fig. 2) is in good agreement with the observational light curves including a shallow decay phase and a normal decay phase (Zhang et al. 2006), because of the energy injection into the ES before $T_{\mathrm{m}}$. More surprisingly, on the other hand, we find that a very similar profile also appears in the TS-dominated cases (e.g., the cases in the left panel of Fig. 2). Please note that the "normal decay" here is actually not produced by the ES. To understand such an unexpected "normal decay", we adopt an approximative dynamic evolution of the TS as $\Gamma_{3} \propto t^{-1 / 2}$ for $t>T_{\mathrm{m}}$, which is extracted from the numerical results shown in Fig. 1. Combining with $n_{4}^{\prime} \propto L_{\mathrm{md}} /\left(\Gamma_{3}^{2} t\right) \propto t^{-2}$, we can get $B_{3}^{\prime} \propto t^{-1 / 2}, \gamma_{\mathrm{m}, 3}^{\prime} \propto t^{1 / 2}$, and $\gamma_{c, 3}^{\prime} \propto t^{1 / 2}$. Then following Sari et al. (1998), we can easily derive $F_{v} \propto t^{-1}$ from the characteristic spectral quantities $v_{\mathrm{m}} \propto t^{0}, v_{c} \propto t^{0}, F_{v, \max } \propto t^{-1}$, and the relationship $v_{\mathrm{m}}<v_{X}<v_{c}$. The ratio of $F_{v} / L_{\mathrm{md}} \propto t$ indicates that the X-ray luminosity of the wind emission does not track the spin-down luminosity of the magnetar with a constant fraction, which is assumed in Yu et al. (2010). However, the more important conjecture proposed by Yu et al. (2010) that some observed X-ray afterglows are directly produced by the magnetar winds can be still strongly favored by the good agreement between the profiles of the TS-dominated afterglow light curves and the observational ones.

Finally, although both the ES- and TS-dominated afterglow light curves are qualitatively consistent with the observations, some tiny differences between them could also be found by some careful statistical studies. For example, the shallow decay phase of the TS-dominated afterglows would be slightly flatter than for the ES-dominated afterglows, and the same is true for the so-called normal decay phase.

\section{Summary}

Following Dai (2004) and Yu \& Dai (2007), we investigated the emission from a TS of a magnetar wind both before and after $T_{\mathrm{m}}$ as well as the simultaneous emission from the GRB ES, where the magnetization of the wind was taken into account. The competition between the emission of the two shocks is mainly determined by the model parameters $\sigma$ and $\epsilon_{\mathrm{e}}$. As a result, an ES-dominated type and a TS-dominated type of afterglows are predicted, both of which are roughly consistent with the observational X-ray afterglows. This indicates that some observed afterglows could come from the wind TS, but not necessarily from the ES. To a certain extent, we regard such TS emission as general internal afterglow emission, although the radius of the TS is nearly as large as that of the ES. To distinguish between these two types of afterglows, some tiny differences in the temporal indices could be useful for some statistical studies such as that in Yu et al. (2010). In this paper, very high values of $\sigma$ were not considered, because in those cases the TS may disappear (Giannios et al. 2008) and the injecting flow is probably dominated by Poynting flux. This was studied thoroughly in Dai \& Lu (1998a,b) and Zhang \& Mészáros (2001).

Acknowledgements. This work is supported by the opening project of the Key Laboratory of Quark and Lepton Physics (MOE) at CCNU (grant no. QLPL2009P01) and by the Self-Determined Research Funds of CCNU (grant no. CCNU09A01020) from the colleges' basic research and operation of MOE of China. X. P. Zheng is supported by the National Natural Science Foundation of China (grant no. 10773004).

\section{References}

Atoyan A. M. 1999, A\&A, 346, L49

Blandford, R. D., \& McKee, C. F. 1976, Phys. Fluids., 19, 1130

Bucciantini, N., Blondin, J. M., Del Zanna, L., \& Amato, E. 2003, A\&A, 405, 617

Bucciantini, N., Quataert, E., Arons, J., et al. 2007, MNRAS, 380, 1541

Bucciantini, N., Quataert, E., Metzger, B. D., et al. 2009, MNRAS, 396, 2038

Campana, S., Antonelli, L. A., Chincarini, G., et al. 2005, ApJ, 625, L23

Coroniti, F. V. 1990, ApJ, 349, 538

Dai, Z. G. 2004, ApJ, 606, 1000

Dai, Z. G., \& Lu, T. 1998a, A\&A, 333, L87

Dai, Z. G., \& Lu, T. 1998b, Phys. Rev. Lett., 81, 4301

Dai, Z. G., \& Lu, T. 2002, ApJ, 565, L87

Dai, Z. G., Wang, X. Y., Wu, X. F., et al. 2006, Science, 311, 1127

Del Zanna, L., Amato, E., \& Bucciantini, N. 2004, A\&A, 421, 1063

Drenkhahn, G. 2002, A\&A, 387, 714

Drenkhahn, G., \& Spruit, H. C. 2002, A\&A, 391, 1141

Emmering, R. T., \& Chevalier, R. A. 1987, ApJ, 321, 334

Fan, Y. Z., Wei, D. M., \& Wang, C. F. 2004, A\&A, 424, 477

Gehrels, N., Chincarini, G., Giommi, P., et al. 2004, ApJ, 611, 1005

Giannios, D. 2008, A\&A, 480, 305

Giannios, D., Mimica, P., \& Aloy, M. A. 2008, A\&A, 478, 747

Huang, Y. F, Gou, L. J., \& Dai, Z. G. 2000, ApJ, 543, 90

Kennel, C. F., \& Coroniti, F. V. 1984, ApJ, 283, 694

Kirk, J. G., \& Skjæraasen, O. 2003, ApJ, 591, 366

Kobayashi, S. 2000, ApJ, 545, 807

Kobayashi, S., \& Sari, R. 2000, ApJ, 542, 819

Liang, E. W., Zhang, B. B., \& Zhang, B. 2007, ApJ, 670, 565

Lyubarsky, Y., \& Kirk, J. G. 2001, ApJ, 547, 437

Medvedev, M. V. 2006, ApJ, 651, L9

Nakar, E. 2007, Phys. Rep., 442, 166

O’Brien, P. T., Willingale, R., Osborne, J., et al. 2006, ApJ, 647, 1213

Rees, M. J., \& Mészáros, P. 1998, ApJ, 496, L1

Rybicki, G. B., \& Lightman, A. P. 1979, Radiative processes in astrophysics (NY: Wiley-Interscience), 163

Sari, R., Piran, T., \& Narayan, R. 1998, ApJ, 497, L17

Shapiro, S. L., \& Teukolsky, S. A. 1983, Black holes, White Dwarfs, and Neutron Stars (NY: John Wiley \& Sons), 278

Woosley, S. E., \& Bloom, J. S. 2006, A\&A, 44, 507

Yu, Y. W., \& Dai, Z. G. 2007, A\&A, 470, 119

Yu, Y. W., Liu, X. W., \& Dai, Z. G. 2007, ApJ, 671, 637

Yu, Y. W., Cheng, K. S., \& Cao, X. F. 2010, ApJ, 715, 477

Zhang, B., \& Kobayashi, S. 2005, ApJ, 628, 315

Zhang, B., \& Mészáros, P. 2001, ApJ, 552, L35

Zhang, B., Fan, Y. Z., Dyks, J., et al. 2006, ApJ, 642, 354

Zhang, D., \& Dai, Z. G. 2008, ApJ, 683, 329 